\title{
Expression and clinical significance of focal adhesion kinase and adrenomedullin in epithelial ovarian cancer
}

\author{
MINGQUN LI ${ }^{1,2}$, LI HONG ${ }^{1}$, MEIMEI LIAO ${ }^{3}$ and GUANGLIN GUO ${ }^{1}$ \\ ${ }^{1}$ Department of Gynecology and Obstetrics, Renmin Hospital of Wuhan University, Wuhan, Hubei 430000; \\ ${ }^{2}$ Department of Gynecology and Obstetrics, Xiangyang No. 1 People's Hospital, Hubei University of Medicine, \\ Xiangyang, Hubei 441100; ${ }^{3}$ Department of Ultrasound Imaging, Renmin Hospital of Wuhan University, \\ Wuhan, Hubei 430000, P.R. China
}

Received July 24, 2014; Accepted April 30, 2015

DOI: $10.3892 / 01.2015 .3278$

\begin{abstract}
The aim of the present study was to investigate the expression of focal adhesion kinase (FAK) and adrenomedullin (ADM) and determine their clinical significance and cooperative role in human epithelial ovarian cancer. The expression of FAK and ADM was investigated in epithelial ovarian cancer, benign ovarian tumors and normal control tissues by immunohistochemical staining and optical microscopy. The FAK and ADM expression and correlation with clinicopathological parameters was analyzed using SPSS 13.0 software. The expression of FAK and ADM in epithelial ovarian cancer was significantly higher compared with that in benign tumors or normal ovarian tissues $(\mathrm{P}<0.01)$; however, no significant difference was observed between benign tumors and normal tissues $(\mathrm{P}>0.05)$. The expression of FAK was found to be correlated with histological grade, clinical stage, lymph node metastasis and prognosis $(\mathrm{P}<0.05)$, but exhibited no significant association with patient age or histological type $(\mathrm{P}>0.05)$. The expression of ADM was significantly correlated with pathological grade, lymph node metastasis and prognosis $(\mathrm{P}<0.05)$, but not with age, clinical stage or histological type $(\mathrm{P}>0.05)$. The Spearman's rank correlation analysis revealed a positive correlation between FAK and ADM expression ( $\mathrm{r}=0.314)$. FAK and ADM were more highly expressed in epithelial ovarian cancer compared with benign tumors or normal ovarian tissues. Furthermore, FAK and ADM may play a cooperative role; specifically, FAK may upregulate ADM in the invasion and migration of epithelial ovarian cancer. Thus, FAK and ADM may represent potential biomarkers for evaluating the malignant potential and prognosis of ovarian cancer.
\end{abstract}

Correspondence to: Professor Li Hong, Department of Gynecology and Obstetrics, Renmin Hospital of Wuhan University, 238 Jiefang Road, Wuhan, Hubei 430000, P.R. China

E-mail: hongliwuhan@163.com

Key words: ovarian cancer, focal adhesion kinase, adrenomedullin

\section{Introduction}

Ovarian cancer is one of the most common malignancies of the female reproductive organs, with an incidence rate second only to cervical cancer. Epithelial tumors account for 50-70\% of primary ovarian tumors, and $85-90 \%$ of ovarian malignancies (1). In clinical treatment, although surgery combined with chemotherapy is an established treatment, the prognosis is poor when ovarian cancer is detected late, with a recurrence rate of up to $85 \%$, and a 5 -year survival rate of $<30 \%$ (2). The pathogenesis of ovarian cancer and the development of new therapeutic strategies, such as endocrine treatment and targeted therapies, are areas of current research (3).

Focal adhesion kinase (FAK) is a $125-\mathrm{kDa}$ non-receptor cytoplasmic tyrosine kinase. Over the last few years, FAK, known as the first intracellular messenger, was found to be involved in cell adhesion, spreading, proliferation, migration and apoptosis (4). Further studies demonstrated that FAK is highly expressed in a number of tumors and is potentially correlated with tumor development and biological behavior (5). Adrenomedullin (ADM) is a 52-amino acid member of the calcitonin family of peptides. ADM is present in a variety of human tissues and participates in a number of pathophysiological processes. Previous studies have indicated that ADM may stimulate tumor development and progression via different pathways (6). To date, little is known regarding the role of FAK and ADM in ovarian cancer. The aim of the present study was to investigate the expression and clinical significance of FAK and ADM in epithelial ovarian cancer.

\section{Materials and methods}

Samples. A total of 60 ovarian tumor samples and 10 normal ovarian tissue samples from patients with other diseases were collected between March, 2008 and May, 2012 at the Department of Gynecology and Obstetrics, Renmin Hospital of Wuhan University (Wuhan, China). The samples were embedded in paraffin and cut in $4-5-\mu \mathrm{M}$ sections. The ovarian tumors comprised 10 benign lesions and 50 epithelial ovarian cancers (20 serous, 17 mucous, 8 endometrioid and 5 other types). The mean patient age was 50.5 years. The malignant tumors were classified according to the International Federation of 
Gynecology and Obstetrics (1988) criteria as early-stage [n=15 (7, stage I; and 8, stage II)] and late-stage [n=35 (28, stage III; and 7 , stage IV)]. Following pathological grading, 32 cases were classified as moderately or highly differentiated and 18 cases as poorly differentiated or undifferentiated.

The 10 cases of benign epithelial ovarian tumors included 7 serous and 3 mucous tumors. The mean patient age was 42 years. The normal ovarian tissue samples were confirmed by pathological examination and the mean age of the patients was 47.3 years. None of the patients had been administered chemotherapy, radiotherapy, hormone therapy or immunotherapy prior to surgery. The patients were followed up until May, 2013.

Written informed consent was obtained from all patients, and the study was approved by the ethics committee of Renmin Hospital of Wuhan University.

Reagents and methods. The rabbit anti-human FAK polyclonal antibody and the streptavidin-peroxidase (SP) kit were purchased from Zhongshan Biotechnology Co. (Beijing China). The rabbit anti-human ADM polyclonal antibody and diaminobenzidine substrate were obtained from Boster Bioengineering Co., Ltd. (Wuhan China). The immunohistochemical SP method was applied according to the manufacturer's instructions. Tissues with known FAK or ADM expression were used as positive control and phosphate-buffered saline instead of primary antibody was used as negative control.

Result evaluation. The tissue sections were evaluated under a microscope (MP5.0-RTV-CLR-10-C, MicroPublisher 5.0; Olympus Inc., Richmond Hill, ON, Canada) and cells with brown granules in the cytoplasm and/or membrane were considered as FAK- or ADM-positive. Semi-quantitative numerical integration was used to determine the positivity level of each section based on the percentage of positive cells and staining intensity. Scores were assigned according to the percentage of positive cells $(0, \leq 5 \% ; 1,6-25 \% ; 2,26-50 \%$; and $3, \geq 51 \%)$ and intensity of staining ( 0 , no staining; 1 , weak; 2 , medium; and 3, strong) in 10 high-magnification fields. In each sample, the scores for positive percentage and staining intensity were added, reflecting different expression levels as follows:,$- \leq 1 ;+, 2-3$; ++, 4-5; and,$+++>5$.

Statistical analysis. Data were analyzed with the $\chi^{2}$ or Fisher's exact tests using SPSS 13.0 software (SPSS, Inc., Chicago, IL, USA) and the groups were compared with the Spearman's rank correlation analysis. Survival data were analyzed with the Kaplan-Meier method, tested with the log-rank method and further analyzed for multiple factors using the Cox regression model. $\mathrm{P}<0.05$ was considered to indicate a statistically significant difference.

\section{Results}

Expression of FAK and ADM in normal, benign and malignant ovarian tissues. (Fig. 1) The FAK protein was primarily expressed in the cytoplasm of cancer cells in $72.0 \%$ (36/50) of ovarian cancer cases and was weakly detected in a limited number of normal and benign cases. The ADM protein was mainly detected in the cytoplasm and/or membrane of cancer
Table I. Expression of FAK and ADM in different ovarian tissues.

\begin{tabular}{lccc}
\hline $\begin{array}{l}\text { Tissue } \\
\text { type }\end{array}$ & $\begin{array}{c}\text { Total } \\
(\mathrm{n}=70)\end{array}$ & $\begin{array}{c}\text { FAK+, no. }(\%) \\
(\mathrm{n}=41)\end{array}$ & $\begin{array}{c}\text { ADM+, no. }(\%) \\
(\mathrm{n}=42)\end{array}$ \\
\hline $\begin{array}{l}\text { Normal } \\
\text { Benign }\end{array}$ & 10 & $2(20.0)$ & $1(10.0)$ \\
Cancer & 10 & $3(30.0)$ & $2(20.0)$ \\
\hline
\end{tabular}

FAK, focal adhesion kinase; ADM, adrenomedullin.

cells, whereas different levels of ADM staining were observed in the stroma and the center of the tumor. The ADM protein was positive in $78.0 \%$ (39/50) of ovarian cancers, 1 normal tissue sample $(+)$ and 2 benign tumors $(+$ and ++$)$. The statistical analysis demonstrated that FAK and ADM were more highly expressed in ovarian cancer compared with normal tissues and benign tumors $(\mathrm{P}<0.05)$, whereas there was no significant difference between normal and benign ovarian tissues $(\mathrm{P}>0.05)$ (Table I).

Expression of FAK and ADM in epithelial ovarian cancer and correlation with clinicopathological parameters. The expression of FAK was significantly correlated with clinical stage, histological grade and lymph node metastasis $(\mathrm{P}<0.05)$; however, there was no significant association with age or histological type $(\mathrm{P}>0.05)$. The expression of ADM was positively correlated with pathological grade and lymph node metastasis $(\mathrm{P}<0.05)$, but not with age, histological type or clinical stage $(\mathrm{P}>0.05)$ (Table II).

Correlation between the expressions of FAK and ADM. The Spearman's rank correlation analysis demonstrated that, among all 50 cases of epithelial ovarian cancer, 31 were positive and 6 were negative for both proteins, suggesting a rank positive correlation between the two $(\mathrm{r}=0.314, \mathrm{P}=0.026)$ (Table III).

Correlation between FAK or ADM expression and prognosis in epithelial ovarian cancer. As demonstrated by the Kaplan-Meier curves (Figs. 2 and 3), FAK- and ADM-positive cases had a significantly lower survival rate compared with cases negative for the two proteins $(\mathrm{P}<0.05)$.

\section{Discussion}

Cell signaling is currently being extensively investigated worldwide. FAK is a critical factor in cell signal transduction pathways and is involved in tumorigenesis, invasion and migration (5). Although ADM is expressed in numerous normal tissues, including the lung, colon, heart, uterus and ovary, it is overexpressed in tumor tissues, including lung cancer, neuroblastoma, gastrointestinal tumors, ovarian cancer and cervical squamous cell carcinoma (6). The present study investigated the effect of FAK and ADM on the invasion and migration of ovarian cancer and analyzed the correlation between their expression and clinicopathological parameters. 
Table II. Correlation between FAK or ADM expression and clinicopathological parameters.

\begin{tabular}{|c|c|c|c|c|c|}
\hline Clinicopathological parameters & $\begin{array}{l}\text { Total } \\
(\mathrm{n}=50)\end{array}$ & $\begin{array}{c}\text { FAK }+, \text { no. }(\%) \\
(n=36)\end{array}$ & P-value & $\begin{array}{l}\mathrm{ADM}+, \text { no. }(\%) \\
\quad(\mathrm{n}=39)\end{array}$ & P-value \\
\hline Age (years) & & & 1.000 & & 1.000 \\
\hline$\leq 50$ & 18 & $13(72.22)$ & & $14(77.78)$ & \\
\hline$>50$ & 32 & $23(71.88)$ & & $25(78.13)$ & \\
\hline FIGO stage & & & 0.017 & & 1.000 \\
\hline $\mathrm{I} / \mathrm{II}$ & 19 & $10(52.6)$ & & $15(78.9)$ & \\
\hline III/IV & 31 & $26(83.9)$ & & $24(77.4)$ & \\
\hline Histological type & & & 0.688 & & 0.646 \\
\hline Mucous & 17 & $11(64.70)$ & & $14(82.35)$ & \\
\hline Serous & 20 & $16(80.00)$ & & $15(75.00)$ & \\
\hline Endometrioid & 8 & $6(75.00)$ & & $7(87.50)$ & \\
\hline Others & 5 & $3(60.00)$ & & $3(60.00)$ & \\
\hline Lymph node metastasis & & & 0.011 & & 0.000 \\
\hline No & 28 & $16(57.1)$ & & $17(60.71)$ & \\
\hline Yes & 22 & $20(90.9)$ & & $22(100.0)$ & \\
\hline Pathological grading (differentiation) & & & 0.000 & & 0.033 \\
\hline Moderate/high & 30 & $16(75.00)$ & & $20(66.7)$ & \\
\hline Poor/undifferentiated & 20 & $20(100.00)$ & & $19(95.0)$ & \\
\hline
\end{tabular}

FAK, focal adhesion kinase; ADM, adrenomedullin; FIGO, International Federation of Gynecology and Obstetrics.
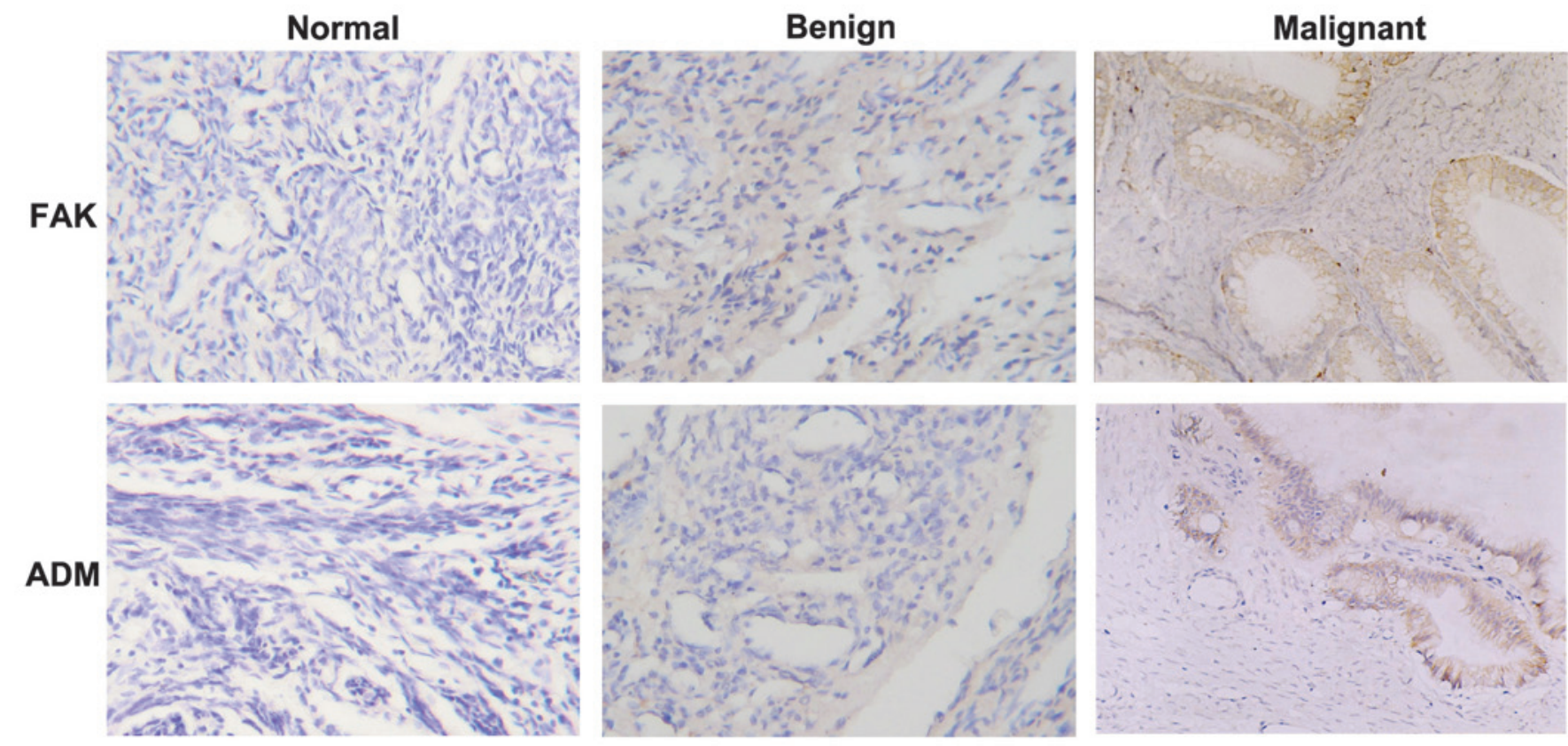

Figure 1. Immunohistochemical staining was used to determine the expression of focal adhesion kinase (FAK) and adrenomedullin (ADM) in normal, benign and malignant ovarian tissues (original magnification, x200).

FAK is mostly expressed in the cytoplasm of ovarian cancer cells. Although cancer as well as paracancerous tissues were positive for FAK expression, the former exhibited a significantly higher level, with the latter exhibiting weak expression in the majority of cases, similar to that observed in normal tissues. Compared with normal and benign ovarian tissues, FAK is overexpressed in malignant ovarian tissues $(\mathrm{P}<0.05)$, exhibiting higher levels in cases with lymph node metastasis or poor differentiation $(\mathrm{P}<0.05)$, whereas its expression was also associated with clinical stage $(\mathrm{P}<0.05)$. Based on these findings, the upregulation of FAK expression may be associated with tumorigenesis, cancer cell proliferation, invasion and migration. As reported by Halder et al (7), the inhibition of FAK phosphorylation may reduce the invasion, migration 
Table III. Rank correlation between FAK and ADM expression.

\begin{tabular}{lccc}
\hline Expression & ADM+ & ADM- & Total \\
\hline FAK + & 31 & 5 & 36 \\
FAK- & 8 & 6 & 14 \\
Total & 39 & 11 & 50 \\
\hline
\end{tabular}

FAK, focal adhesion kinase; ADM, adrenomedullin.

and spreading ability of cancer cells; in addition, suppression of FAK expression may restore the sensitivity of ovarian cancer cells to drug therapy. FAK is a critical regulator of cellular signaling pathways essential to cell survival and division and its overexpression in epithelial and mesenchymal tumors suggests that FAK may play a role in cell invasion and mobility (8). In normal cells, FAK may serve as a sensor of cell adhesion, which may inhibit anchorage-dependent cell growth (9); however, the overexpression of FAK in transformed or cancer cells may relieve this inhibition, resulting in cell overgrowth without adhesion or anchorage. It was reported that FAK expression was increased in the majority of malignant tumors, including colon, thyroid, prostate and brain cancers, but weakly expressed in benign tumors $(10,11)$. Sood et al (12) found that the protein level of FAK in ovarian adenocarcinoma is 4-fold higher compared with that in normal ovarian tissues; their results were consistent with those of other previous studies (13). However, no differences in FAK expression have yet been described in tumor cells from different patients. Our findings indicate that significantly high levels of FAK in ovarian cancer may be an independent indicator of poor prognosis.

The present study also demonstrated a significant difference in ADM expression between ovarian cancer and normal/benign ovarian tissues $(\mathrm{P}<0.05)$. In ovarian cancer cases, no staining for ADM was observed in non-tumor areas, suggesting that ADM was produced specifically by ovarian cancer cells. As regards clinicopathological parameters, the ADM expression was correlated with differentiation level and lymph node metastasis, but not with age, histological type or clinical stage, which was consistent with the findings of Frede et al (14). The intensity and localization of ADM staining were associated with tumor differentiation. In poorly differentiated or undifferentiated samples, ADM staining was strong and evenly distributed; in samples exhibiting moderate or high differentiation, ADM staining was weak and mainly localized at the periphery or along the border of cancer foci. These results suggest that poorly differentiated ovarian cancer may grow rapidly and invade actively and aggressively, whereas moderately or highly differentiated ovarian cancer exhibits a lower malignant potential. ADM is involved in cell proliferation, angiogenesis and immunosuppression (15). Increased expression of ADM in cancer cells may result in aggressive tumor growth, in turn producing more ADM (16). Ovarian cancers exhibiting moderate or high differentiation are relatively less malignant and the high ADM levels may help the cancer cells to spread, which is consistent with the localization of ADM staining at the periphery or along the

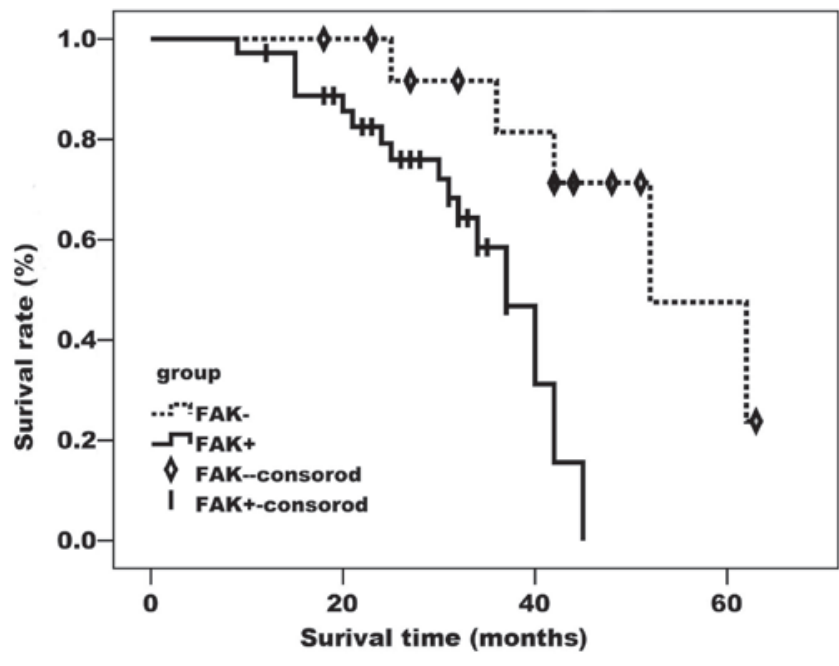

Figure 2. Kaplan-Meier survival curve for focal adhesion kinase (FAK)-positive and -negative ovarian cancer. FAK-positive cases had an average survival time of 34.1 months. No patients survived for $>5$ years. FAK-negative cases had an average survival time of 52.1 months and a 5-year survival rate of $47.8 \%$. FAK-positive cases exhibited a significantly lower survival rate compared with FAK-negative cases $\left(\log\right.$-rank $\left.\chi^{2}=8.316, \mathrm{P}=0.004\right)$.

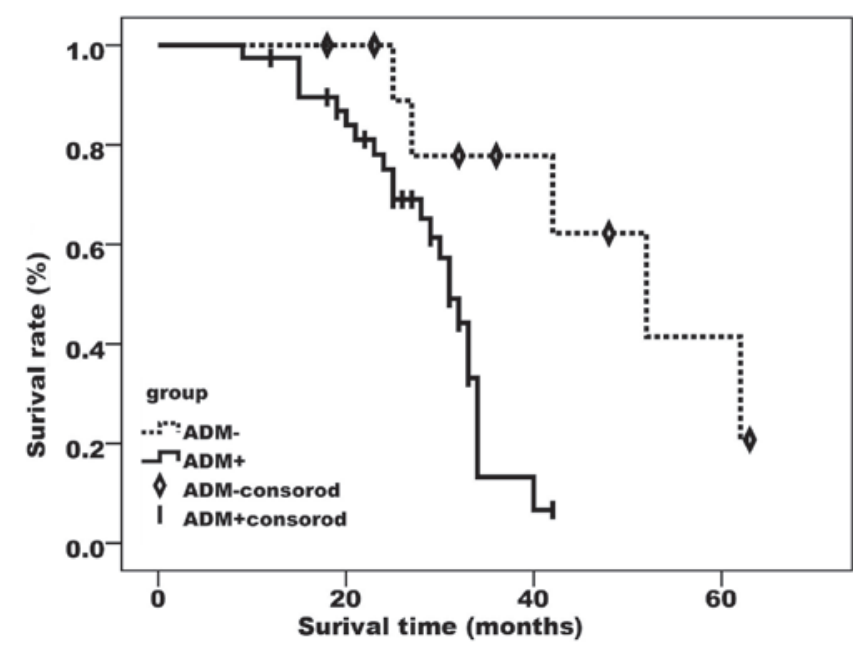

Figure 3. Kaplan-Meier survival curve for adrenomedullin (ADM)-positive and -negative ovarian cancer. ADM-positive cases had an average survival time of 29.4 months. No patients survived for $>5$ years. ADM-negative cases had an average survival time of 49.0 months and a 5-year survival rate of 42.5\%. ADM-positive cases exhibited a significantly lower survival rate compared with ADM-negative cases $\left(\log -\operatorname{rank} \chi^{2}=7.758, \mathrm{P}=0.005\right)$.

border of cancer foci (17). This morphological characteristic suggests an association between tumor behavior and ADM expression: Increased malignancy correlates with higher ADM levels. Therefore, ADM may be an important determinant and marker of aggressive tumor behavior.

The Spearman's rank correlation analysis demonstrated that there is a positive correlation between the expressions of FAK and ADM. As a key component of the signaling pathways triggered by integrins, FAK is a kinase involved in cell carcinogenesis, differentiation and metastasis and is also involved in normal cell transformation and development, adhesion, proliferation and apoptosis (18). Overexpression of FAK may result in the dysregulation of ADM expression. It 
was reported that ADM is a mediator in the crosstalk between mast and tumor cells, a stimulator of angiogenesis and mitosis of tumor cells and an apoptosis inhibitor of vascular endothelial cells $(19,20)$. Therefore, ADM may have a critical function in tumor development and metastasis.

In conclusion, FAK and ADM are involved in cell invasion and migration, which are characteristic features of malignant tumors and the main causes of cancer-related mortality. The present study demonstrated the potential value of FAK and ADM as biomarkers in epithelial ovarian cancer. FAK and ADM may be useful for the early diagnosis of high-risk patients, determination of malignant potential, assessment of prognosis and treatment design. FAK and ADM may also be investigated as potential therapeutic targets for the inhibition of tumor invasion and metastasis.

\section{References}

1. Morgan RJ Jr, Alvarez RD, Armstrong DK, Boston B, Burger RA, Chen LM, Copeland L, Crispens MA, Gershenson D, Gray HJ, et al; National Comprehensive Cancer Network: Epithelial ovarian cancer. J Natl Compr Canc Netw 9: 82-113, 2011.

2. Jemal A, Siegel R, Xu J and Ward E: Cancer statistics, 2010. CA Cancer J Clin 60: 277-300, 2010.

3. Colombo N, Peiretti M, Parma G, et al: Newly diagnosed and relapsed epithelial ovarian carcinoma: ESMO Clinical Practice Guidelines for diagnosis, treatment and follow-up. Ann Oncol 21: v23-v30, 2010

4. Baron V, Calléja V, Ferrari P, Alengrin F and Van Obberghen E: p125Fak focal adhesion kinase is a substrate for the insulin and insulin-like growth factor-I tyrosine kinase receptors. J Biol Chem 273: 7162-7168, 1998.

5. McLean GW, Carragher NO, Avizienyte E, Evans J, Brunton VG and Frame MC: The role of focal-adhesion kinase in cancer - a new therapeutic opportunity. Nat Rev Cancer 5: 505-515, 2005.

6. Nikitenko LL, Leek R, Henderson S, et al: The G-protein-coupled receptor CLR is upregulated in an autocrine loop with adrenomedullin in clear cell renal cell carcinoma and associated with poor prognosis. Clin Cancer Res 19: 5740-5748, 2013.

7. Halder J, Landen CN, Lutgendorf SK, et al: Focal adhesion kinase silencing augments docetaxel-mediated apoptosis in ovarian cancer cells. Clin Cancer Res 11: 8829-8836, 2005.
8. Huang YT, Lee LT, Lee PP, Lin YS and Lee MT: Targeting of focal adhesion kinase by flavonoids and small-interfering RNAs reduces tumor cell migration ability. Anticancer Res 25: 2017-2025, 2005.

9. Gao M, Liu X, Sun H, Ren H, Wang L and Shen Y: Study on FAK regulation of migration of vascular endothelial cells depending upon focal adhesion proteins. Sheng Wu Yi Xue Gong Cheng Xue Za Zhi 30: 567-571, 2013 (In Chinese).

10. Grisaru-Granovsky S, Salah Z, Maoz M, Pruss D, Beller U and Bar-Shavit R: Differential expression of protease activated receptor 1 (Parl) and pY397FAK in benign and malignant human ovarian tissue samples. Int J Cancer 113: 372-378, 2005.

11. Saldanha SN and Tollefsbol TO: Pathway modulations and epigenetic alterations in ovarian tumorbiogenesis. J Cell Physiol 229: 393-406, 2014.

12. Sood AK, Coffin JE, Schneider GB, Fletcher MS, DeYoung BR, Gruman LM, Gershenson DM, Schaller MD, Hendrix MJ: Biological significance of focal adhesion kinase in ovarian cancer: role in migration and invasion. Am J Pathol 165: 1087-1095, 2004.

13. Judson PL, He X, Cance WG and Van Le L: Overexpression of focal adhesion kinase, a protein tyrosine kinase, in ovarian carcinoma. Cancer 15: 1551-1556, 1999.

14. Frede S, Freitag P, Otto T, Heilmaier C and Fandrey J: The proinflammatory cytokine interleukin 1beta and hypoxia cooperatively induce the expression of adrenomedullin in ovarian carcinoma cells through hypoxia inducible factor 1 activation. Cancer Res 65: 4690-4697, 2005.

15. Cuttitta F, Pío R, Garayoa M, Zudaire E, Julián M, Elsasser TH, Montuenga LM and Martínez A: Adrenomedullin functions as an important tumor survival factor in human carcinogenesis. Microsc Res Tech 57: 110-119, 2002.

16. Zudaire E, Martinez A and Cuttitta F: Adrenomedullin and cancer. Regul Pept 112: 175-183, 2003.

17. Pang X, Shang H, Deng B, Wen F and Zhang Y: The Interaction of Adrenomedullin and Macrophages Induces Ovarian Cancer Cell Migration via Activation of RhoA Signaling Pathway. Int J Mol Sci 14: 2774-2787, 2013.

18. Provenzano PP and Keely PJ: The role of focal adhesion kinase in tumor initiation and progression. Cell Adh Migr 3: 347-350, 2009.

19. Karpinich NO, Hoopes SL, Kechele DO, Lenhart PM and Caron KM: Adrenomedullin function in vascular endothelial cells: Insights from genetic mouse models. Curr Hypertens Rev 7: 228-239, 2011

20. Zudaire E, Martínez A, Garayoa M,Pío R, Kaur G, Woolhiser MR, Metcalfe DD, Hook WA, Siraganian RP, Guise TA, et al: Adrenomedullin is a cross-talk molecule that regulates tumor and mast cell function during human carcinogenesis. Am J Pathol 168: 280-291, 2006. 